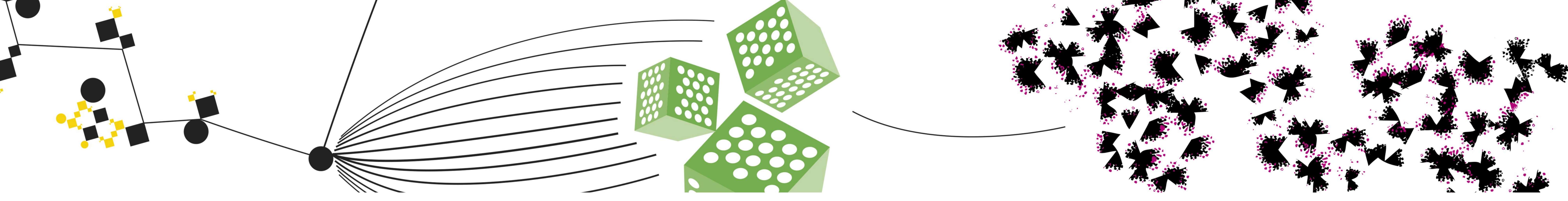

\title{
5G Wideband Magneto-Electric Dipole Antenna Fed by a Single- Layer Corporate-Feed Network based on Ridge Gap Waveguide
}

\section{Wai Yan Yong ${ }^{1}$, Thomas Emanuelsson ${ }^{2,3}$, Andrés Alayón Glazunov ${ }^{1,3}$}

1. Radio System Group, University of Twente, Enschede, Netherlands.

2. Gapwaves AB, Gothenburg, Sweden.

3. Chalmers University of Technology, Gothenburg, Sweden.

\section{Design of MED}

The radiating elements is realized by the four metallic pins and the cavity walls surrounding them.

The top surface of 4 metallic pins equivalent electric dipole

- Gaps between the metallic pins and cavity wall : equivalent magnetic dipole

\section{Ridge Gap Waveguide}

> Controlling the propagation of waves using parallel plate waveguide concept and with the helps of AMC pins

AMC pins provides the band-stop characteristics and determined the operating band of the RGW.

- Main Advantages: No good electrical contact is required for multilayer waveguide structure $\rightarrow$ relax the fabrication cost
Simulated S-11 and Directivity

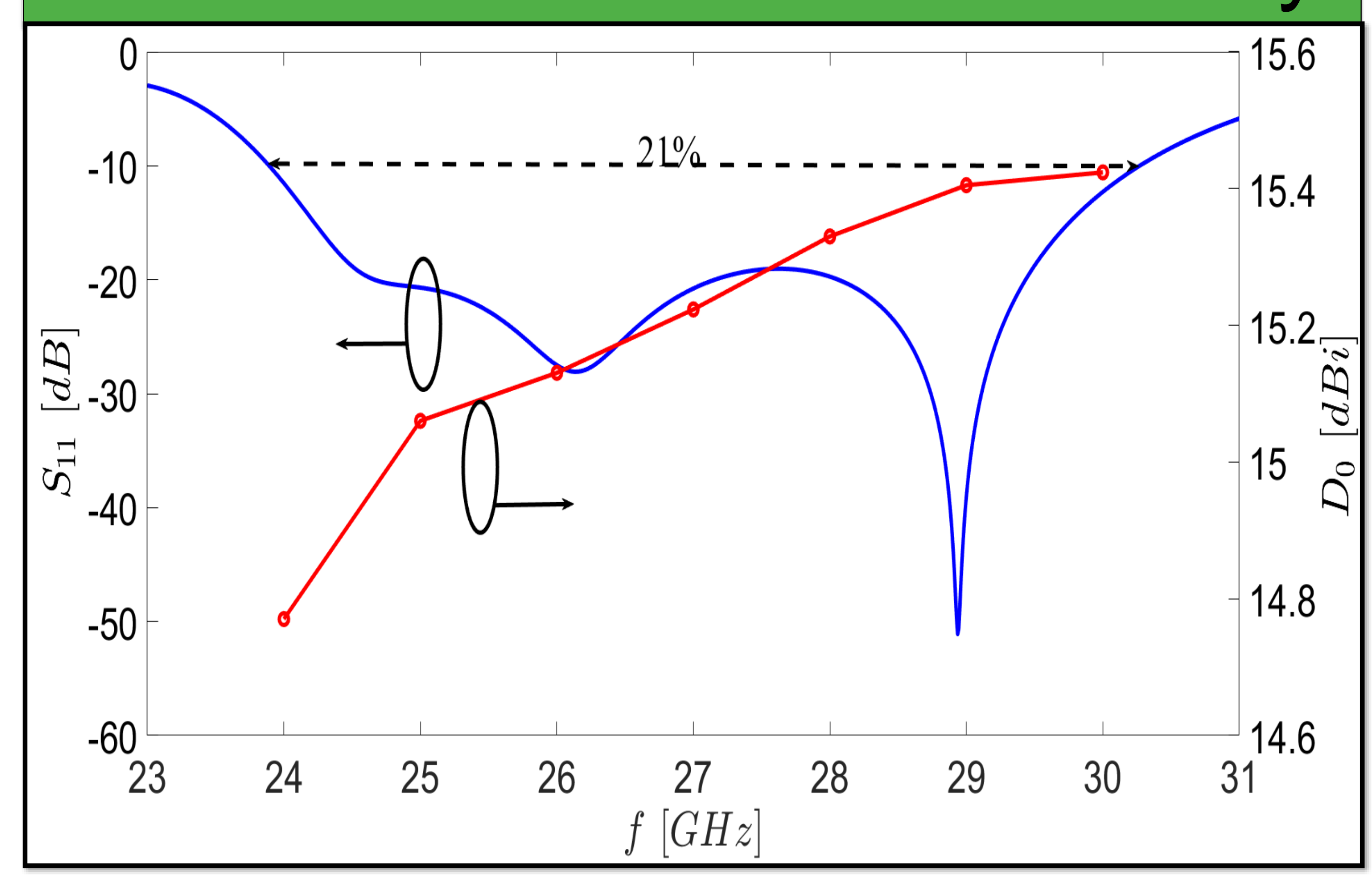

\section{Dispersion diagram of the} proposed RGW

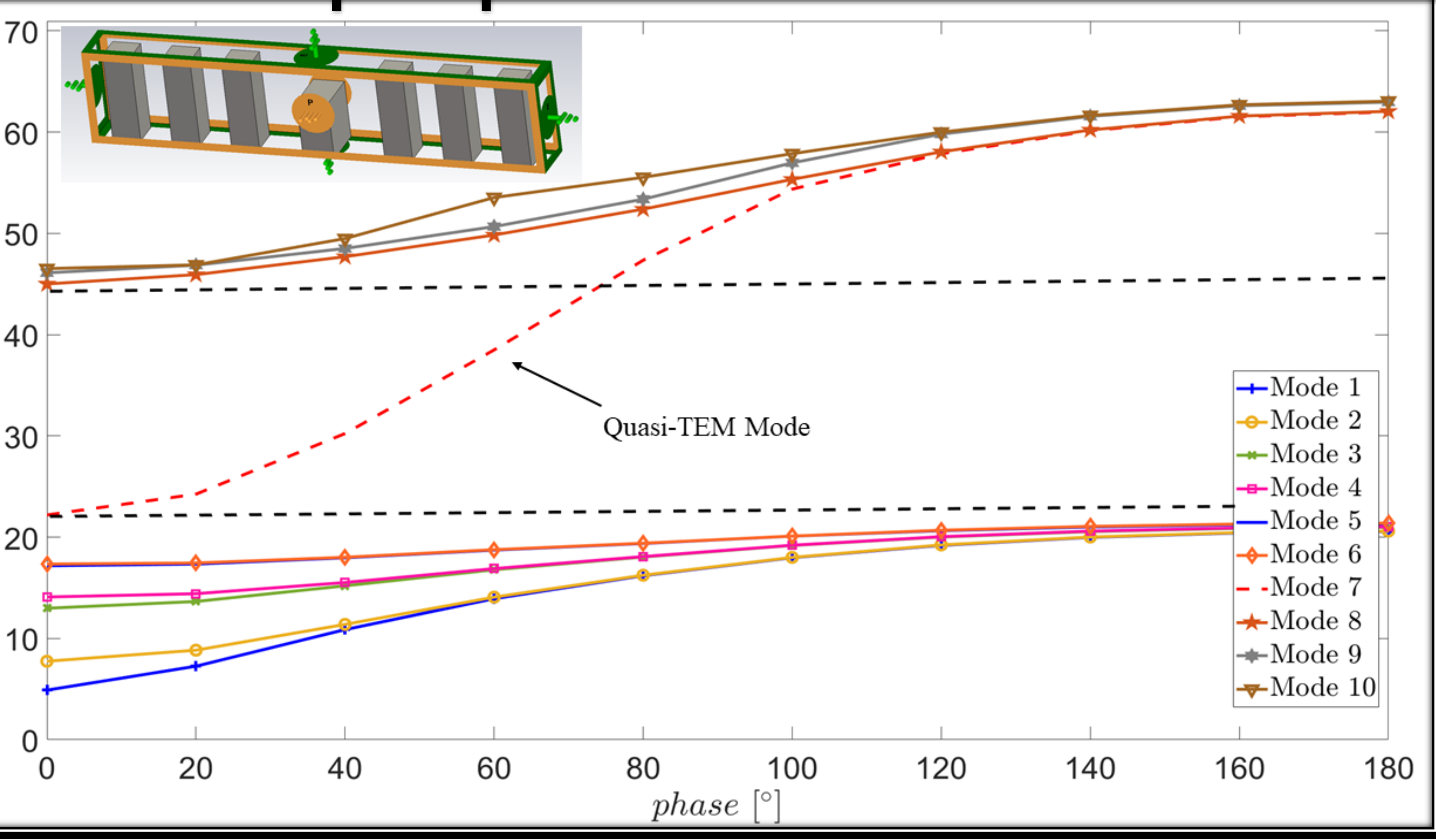

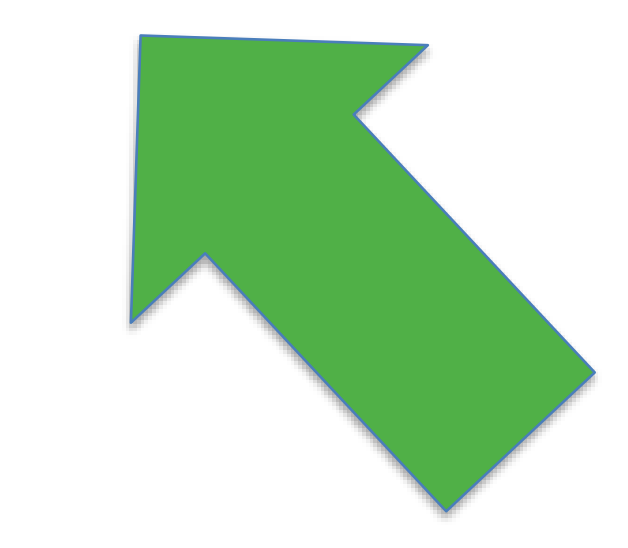
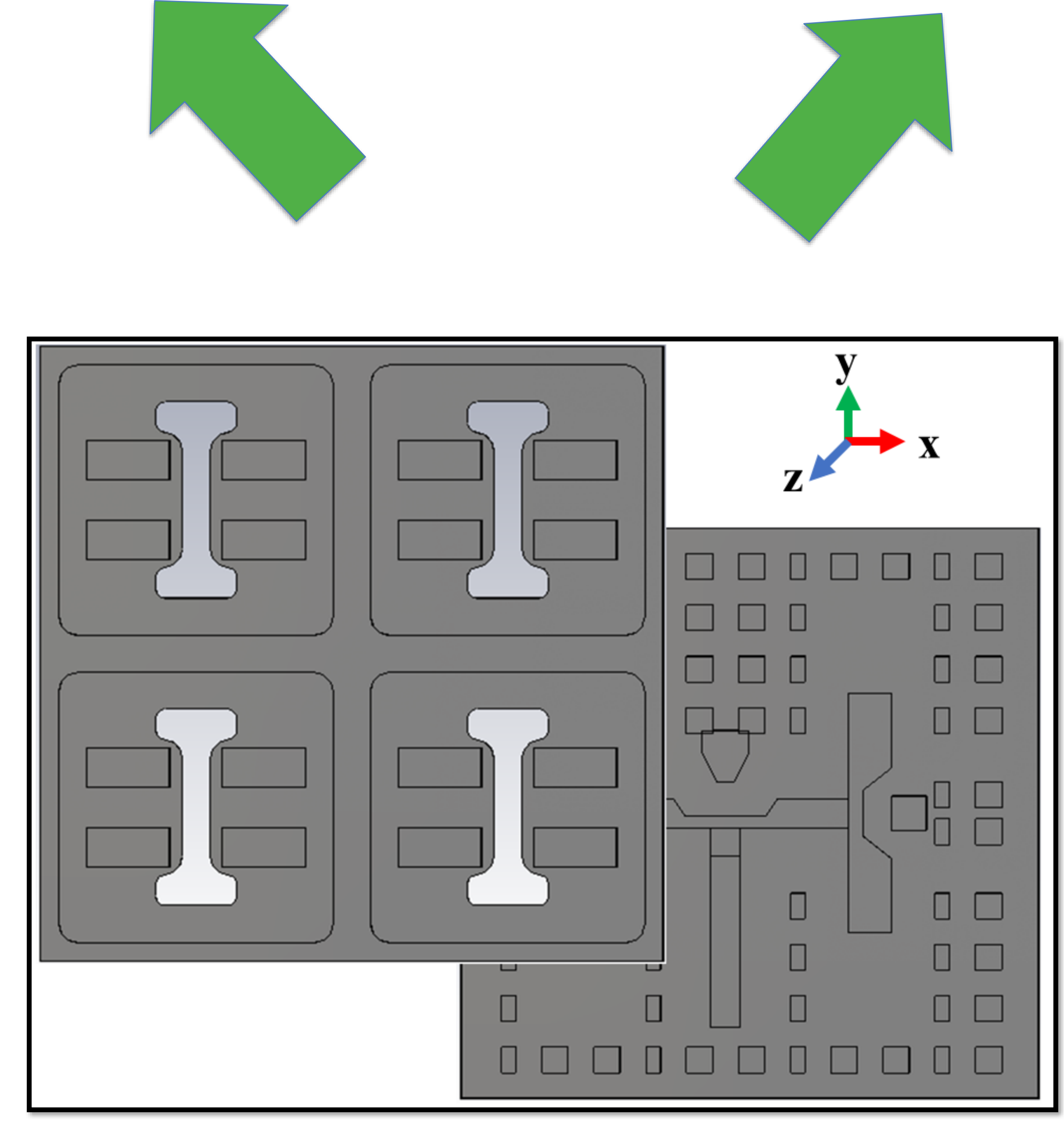

\section{Acknowledgment}

This project has received funding from the European Union Horizon 2020 research and innovation program under the Marie Sklodowska-Curie grant agreement No. 766231 WAVE-COMBE H2O20MSCA-ITN-2017.

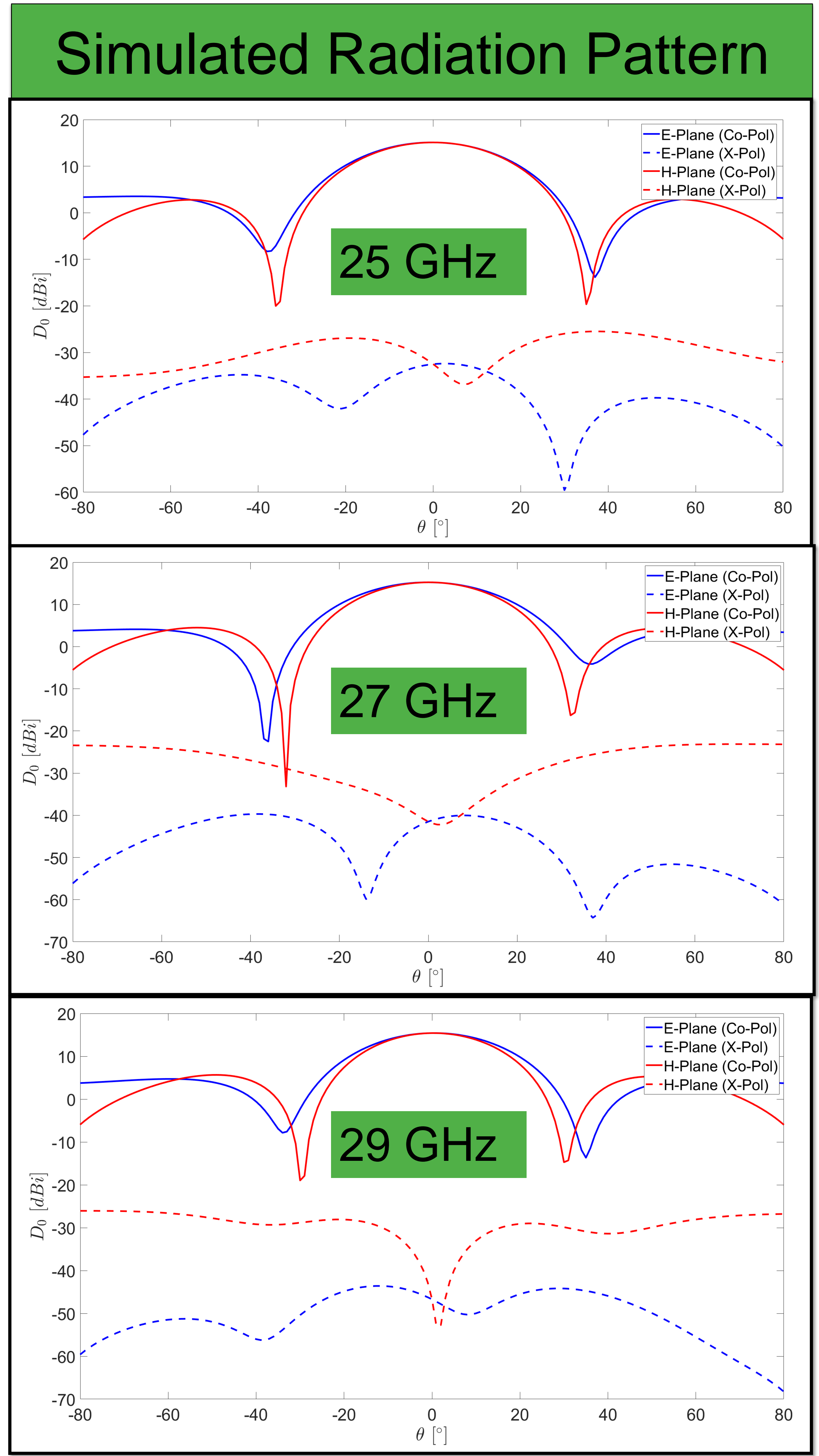

\section{'^APWAVES UNIVERSITY OF TWENTE.}

